\title{
Adaptive Closed-Loop Power Control Using an MMSE Receiver in DS-CDMA Systems*
}

\author{
Lian Zhao and Jon W Mark \\ Centre for Wireless Communications \\ Electrical and Computer Engineering Department \\ University of Waterloo
}

\begin{abstract}
A closed-loop power control algorithm, which includes both power control and power allocation functions for DS-CDMA systems, is proposed. The target power level is updated by an iterative algorithm for a minimum mean square error (MMSE) linear filter receiver. The received power is compared with the target to generate the current power control command (PCC). The transmitted power is adjusted by applying a variable stepsize generator that utilizes the PCC history and the estimated mobile speed. Simulation results are presented to show the improvement of the proposed adaptive power control over the conventional fixed stepsize power control.
\end{abstract}

\section{Introduction}

Power control is one of the most important factors in DS-CDMA systems. Transmit power control (TPC) aims to compensate for the power fluctuations between the transmitter and the receiver so as to maintain the received power at a desired level. TPC is performed by using an open loop (OLPC) and a closedloop (CLPC) strategy. OLPC attempts to eliminate the slowly varying factors such as path loss and shadowing effects. CLPC is used to compensate for a wide range of fast fading effects. Our research is focused on using the CLPC to mitigate Rayleigh fading $[1,2]$. The first research objective, therefore, is to minimize the standard deviation (STD) of the received power by using TPC.

Conventional approach for TPC is to use a sequence of 1 bit power control command $(\mathrm{PCC})$ to regulate the mobile's transmit power using a fixed stepsize. It has been investigated in $[3,4]$ that there exists an optimal stepsize in terms of tracking ability for a given mobile speed. An improvement is made in [5],

\footnotetext{
* This work has been supported by the Natural Science and Engineering Council of Canada under grant no. RGPIN 7779.
} 
where a different stepsize is used for different mobile speed. Another case of the variable stepsize approach is the inverse update algorithm [6], where the stepsize at each iteration is made equal to the inverse of the estimated channel fade. This algorithm offers better performance at the expense of complexity and increased bandwidth requirements on the downlink to carry the extra PCC bits. All the above schemes belong to the one-step TPC. The shortcoming with the one-step TPC is that the PCC is discarded when the update is executed. Thus, it fails to explore the correlations of the PCC history. The inclusion of the PCC history (multi-step) will generally enhance the tracking ability. Adaptive stepsize based on a fixed look-up table using the most recent several PCC has been proposed in [1]. The shortcoming of [1] is that the selected stepsize is not related to the mobile speed.

In this paper, we propose an adaptive multi-step CLPC approach, which achieves adaptive stepsize by using PCC history and the estimated mobile speed. The advantages are (1) PCC history is exploited for multi-step power control; (2) the stepsizes are associated with the estimated mobile speed; (3) PCC is still one bit each time, no extra PCC bit is required; (4) better tracking ability is achieved. The STD of the received power is greatly reduced with a negligible increase in computing.

Besides TPC, power control has also been used as an efficient approach for resource management by way of "power allocation" [7]-[9]. By employing the advanced receiver structures, the target power can be reduced considerably, leading to a lowered transmit power and prolonged battery life. We consider using a Minimum Mean Squared Error (MMSE) linear receiver [10] to demodulate the desired signal in a multiple access environment. An algorithm [11] which iteratively updates the target powers and receiver filter coefficients is applied for the purpose to reduce the target power.

\section{Outer Loop Power Control Algorithm}

Consider a synchronous CDMA system with a processing gain $N$, and BPSK modulation. $N$-dimensional column vectors $\mathbf{s}_{i}$ and $\mathbf{c}_{i}$ are used to denote the preassigned unique signature sequence and the linear receiver filter coefficients of user $i$, respectively. The received signal at the base station is

$$
\mathbf{r}_{i}=\sum_{j=1}^{K} \sqrt{p_{j} h_{j}} b_{j} \mathbf{s}_{j}+\mathbf{V}
$$

where $K$ is the number of users in the system, $b_{j}, p_{j}$ and $h_{j}$ are the information bit, the transmitted power, and the channel gain of user $j$, respectively. The term $\mathbf{V}=\left(v_{1}, \ldots, v_{N}\right)^{t}$ is an $N$-dimensional white Gaussian noise vector with per-component zero-mean and variance $E\left[v_{l}^{2}\right]=\sigma^{2}$. The receiver filter output 
of user $i$ is

$$
y_{i}=\sum_{j=1}^{K} \sqrt{p_{j} h_{j}} b_{j}\left(\mathbf{c}_{i}^{t} \mathbf{s}_{j}\right)+n_{i}
$$

where $n_{i}=\mathbf{c}_{i}^{t} \mathbf{V}$ is a Gaussian random variable with zero mean and variance $\sigma^{2} \mathbf{c}_{i}^{t} \mathbf{c}_{i}$. The signal to interference ratio (SIR) of user $i$ can be written as

$$
S I R_{i}=\frac{p_{i} h_{i}\left(\mathbf{c}_{\mathbf{i}}^{t} \mathbf{s}_{\mathbf{i}}\right)^{2}}{\left(\mathbf{c}_{\mathbf{i}}^{t} \mathbf{c}_{\mathbf{i}}\right) \sigma^{2}+\sum_{j \neq i} p_{j} h_{j}\left(\mathbf{c}_{\mathbf{i}}^{t} \mathbf{s}_{\mathbf{j}}\right)^{2}}
$$

Our aim is to find optimal powers, $\mathbf{p}=\left[p_{1}, p_{2}, \cdots, p_{K}\right]$, and the filter coefficients, $\mathbf{c}_{i}$, for $i=1, \cdots, K$, such that the total power is minimized while each user $i$ satisfies the quality of service requirement, i.e., $S I R_{i} \geq \gamma_{i}^{*}$, where $\gamma_{i}^{*}$ is the target SIR for user $i$.

By using the analysis of standard interference functions [11]-[13], an iterative algorithm for user $i$ can be written as [11]

$$
\begin{aligned}
\mathbf{c}_{i}(n) & =\sqrt{p_{i}}\left(1+p_{i} \mathbf{s}_{1}^{t} \mathbf{A}_{i}(\mathbf{p}(n))^{-1} \mathbf{s}_{i}\right)^{-1} \mathbf{A}_{i}^{-1}(\mathbf{p}(n)) \mathbf{s}_{i} \\
p_{i}(n+1) & =\frac{\gamma_{i}^{*}}{h_{i}}\left[\frac{\sum_{j \neq i} p_{j}(n) h_{j}\left(\mathbf{c}_{i}^{t}(n) \mathbf{s}_{j}\right)^{2}+\left(\mathbf{c}_{i}^{t}(n) \mathbf{c}_{i}(n)\right) \sigma^{2}}{\left(\mathbf{c}_{i}^{t}(n) \mathbf{s}_{i}\right)^{2}}\right]
\end{aligned}
$$

where $\mathbf{A}$ is a function of the powers and signature sequences of the interferers, and is given as $\mathbf{A}_{i}=\sum_{j \neq i} p_{j} h_{j} \mathbf{s}_{j} \mathbf{s}_{j}^{t}+\sigma^{2} \mathbf{I}$, where $\mathbf{I}$ is an identity matrix.

\section{Inner Loop Power Control Algorithm}

Fig. 1 shows the log-linear model of the proposed CLPC. The transmit power $P_{t}(n-1) \mathrm{dB}$ is updated by $\Delta(n-1) \mathrm{dB}$ each $T_{p}$ seconds, which is called the power control cycle, to obtain the transmit power level $P_{t}(n)$ at time instant $n$,

$$
P_{t}(n)=P_{t}(n-1)+m(n-1-k) * \Delta(n-1),
$$

where $m(n-1-k)$ is PCC bit $( \pm 1)$, and the index $k$ is the number of loop delay which accounts for generating, transmitting, and executing the PCC. The corresponding received signal power at the base station is $P_{r}(n)=P_{t}(n)+$ $x(n)$, where $x(n)$ is the fading gain. The received signal power $P_{r}(n)$ and the receiver filter coefficients $\mathbf{c}^{*}(n)$ are used to generate the desired power level $P^{*}(n)$ by the functional block C2P, which is an implementation of (5). Then, the desired power level $P^{*}(n)$ is fed back to the functional block P2C by using (4) to update $c^{*}(n)$, which will be used in the next power control cycle. The received power $P_{r}(n)$ is then compared with the desired level $P^{*}(n)$, and a $P C C$ is generated and fed back to the mobile. The model in Fig. 1 also includes the possibility of return channel errors and loop delay $k T_{p}$. 


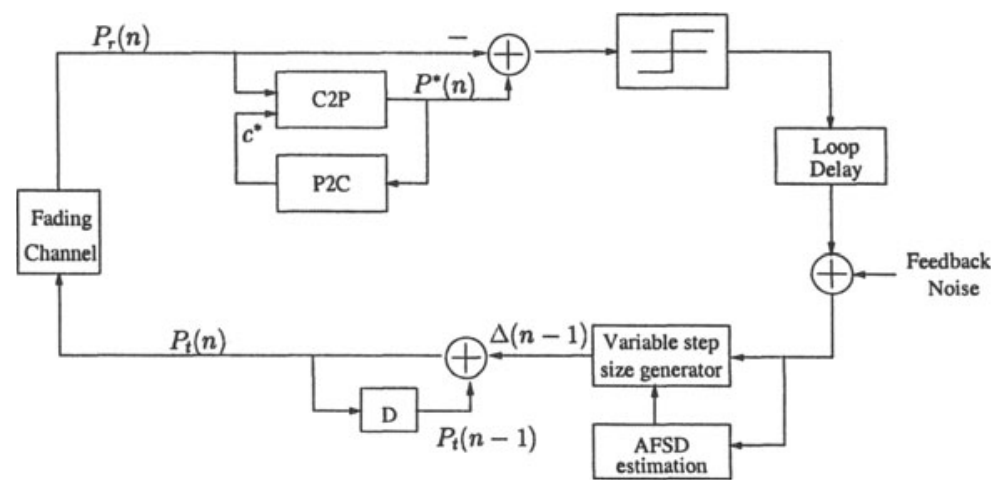

Figure 1. Proposed power control model.

The working principle of the proposed adaptive stepsize is to apply variable stepsize based on PCC bit pattern, which can be written as

$$
\mathbf{m}=[m(1), m(2), \ldots, m(L), \ldots, m(L+k)],
$$

where $m(L+k)$ is the most recent PCC generated at the base station. Due to the loop delay of $k T_{p}$, the most recent PCC bit is $m(L)$ at the mobile. For one-step power control, only $m(L)$ is used for transmit power updating; while for the proposed multi-step adaptive power control, the contents from $m(L)$ down to $m(1)$ and the estimated mobile speed are used to generate the applied stepsize. The length $L$ is called the PCC memory length. After execution of the transmit power updating, $m$ is shifted one bit to the left for the next cycle.

Table 1 is an example of the stepsize look-up table when the mobile speed is $40 \mathrm{~km} / \mathrm{h}$. The PCC memory length is set at 7 . Thus, the column $m(7)$ is the most recent PCC bit at the mobile, and $m(1)$ is the oldest PCC. An " $X$ " represents "don't care". When a "- 1 " is received, it is compared with the previous PCC. If the previous bit is a "-1", then the comparison continues, until a " +1 " is reached or until all the bits in the memory have been compared. The index of the applied stepsize is determined by the number of consecutive " 1 "s counted. The look-up table for a " +1 " appearing in the column of $m(7)$ is not presented due to assumed symmetry.

The variable stepsize $\delta(1)$ to $\delta(7)$ in Table 1 is obtained by taking statistical average over fading processes, i.e., $\delta(1)$ is the average of the fading gain difference between the lowest fade and the second lowest fade, and so on. When executing TPC, further improvement can be achieved by adjusting these average stepsizes appropriately. More results and explanations will be presented in the simulation section.

In order to estimate mobile speed, we define a parameter, namely, the Average Fading Slope Duration (AFSD), as the statistical average number of PCC 
during the period when the fade changes from its peak to the valley, or from the valley to the peak. Given the power control frequency, the value of the AFSD is directly related to the mobile speed. Simulated AFSD is shown in Table 2 for various mobile speed. For all the simulated results throughout the paper, the carrier frequency is set at $900 \mathrm{Mhz}$, and power control cycle is 1.25 $\mathrm{ms}(800 \mathrm{~Hz})$. When the mobile needs to estimate the speed, the mobile may transmit a constant power level around the target regardless of the PCC. Then the mobile counts the consecutive number of +1 or -1 PCC. The resultant average number can be regarded as the average AFSD. The mobile speed can thus be approximately estimated from Table 2 .

Table 1. Look-up table to generate variable stepsize when mobile speed is $40 \mathrm{~km} / \mathrm{h}$.

\begin{tabular}{cccccccccc}
\hline$m(1)$ & $m(2)$ & $m(3)$ & $m(4)$ & $m(5)$ & $m(6)$ & $m(7)$ & stepsize $\delta(i)$ & adjusted $\delta(i)$ & index \\
\hline & & & & $\mathrm{X}$ & +1 & -1 & -1.38 & -1.35 & 1 \\
& & & $\mathrm{X}$ & +1 & -1 & -1 & -1.40 & -1.0 & 2 \\
& $\mathrm{X}$ & $\mathrm{X}$ & +1 & -1 & -1 & -1 & -1.01 & -0.74 & 3 \\
$\mathrm{X}$ & +1 & -1 & -1 & -1 & -1 & -0.74 & -0.52 & 4 \\
+1 & -1 & -1 & -1 & -1 & -1 & -1 & -0.52 & -0.37 & 5 \\
-1 & -1 & -1 & -1 & -1 & -1 & -1 & -0.37 & -0.28 & 6 \\
\hline
\end{tabular}

Table 2. Simulated AFSD, carrier frequency $900 \mathrm{Mhz}$, PC frequency $800 \mathrm{~Hz}$.

\begin{tabular}{cccccccccc}
\hline Speed $(\mathrm{km} / \mathrm{h})$ & 10 & 15 & 20 & 25 & 30 & 35 & 40 & 45 & 50 \\
\hline AFSD & 19.40 & 17.65 & 14.88 & 12.17 & 10.48 & 9.15 & 7.97 & 7.22 & 6.46 \\
\hline
\end{tabular}

\section{Simulation Results}

Fig. 2 shows the target power generated using the MMSE iterative algorithm. Assume that the system supports voice, video and data services, with target SIR being $\{6,8,10\} \mathrm{dB}$, and number of users $\{10,5,5\}$, respectively. The AWGN noise power level is $\sigma^{2}=10^{-4}$. Each user is allocated a Gold sequence with length 31 . The applied polynomials for the Gold sequence are 24 and 35 in octal notations. At the beginning of the iterations, the transmit power is set at $13 \mathrm{~dB}$ below the AWGN noise power, and the filter coefficients are initialized to be the signature sequences of the users. It can be seen that the power converges after 3 iterations.

Fig. 3 is an illustration of the fixed stepsize power control, where the target power level is determined by the outer loop power control. Here, one of the 


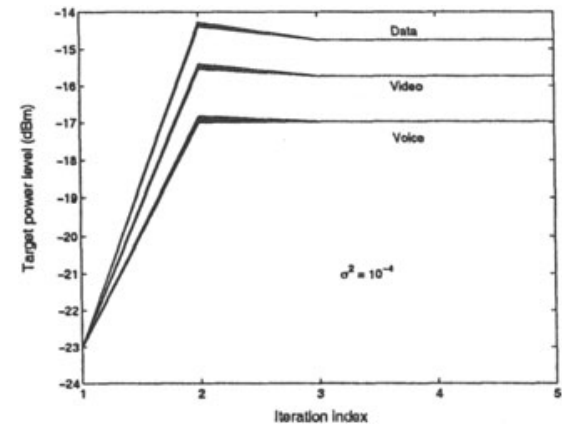

Figure 2. Target power levels for the MMSE iteration algorithm.

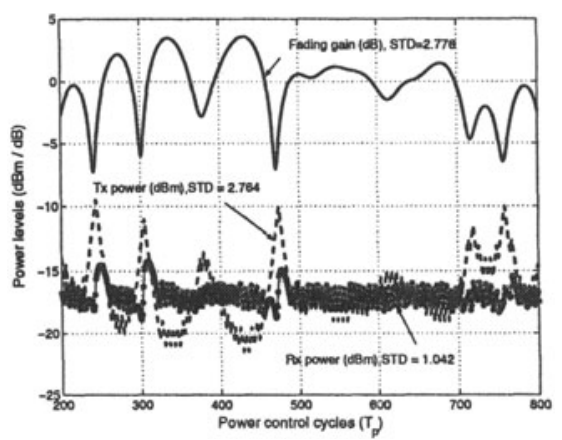

Figure 3. Illustration of the fixed stepsize power control.

voice users is assumed to be the desired user. The parameter values used are: mobile speed equals $10 \mathrm{~km} / \mathrm{h}$, stepsize equals $0.5 \mathrm{~dB}$, no delay and no random errors. Under these conditions, we can see that the CLPC with fixed stepsize $0.5 \mathrm{~dB}$ works quite well. The received power STD is reduced to 1.042 by TPC. Simulated optimal stepsize to minimize the STD of the received power is 0.5 $\mathrm{dB}$ for mobile speed $5-10 \mathrm{~km} / \mathrm{h}, 0.75 \mathrm{~dB}$ for $15-35 \mathrm{~km} / \mathrm{h}, 1 \mathrm{~dB}$ for $40-45 \mathrm{~km} / \mathrm{h}$, and $0.75 \mathrm{~dB}$ for $50 \mathrm{~km} / \mathrm{h}$.

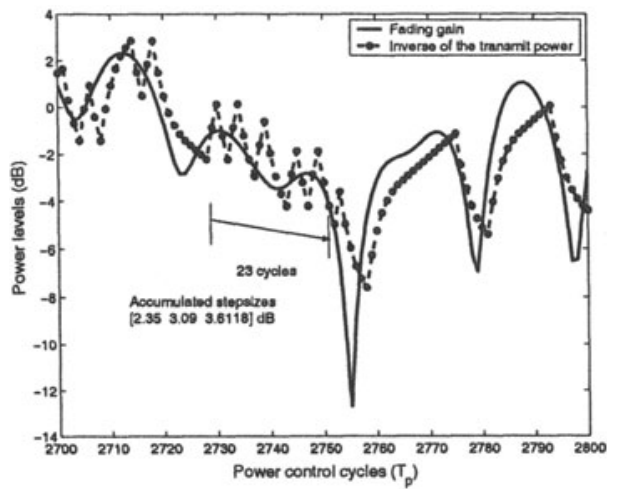

(a) Profile for adaptive stepsize

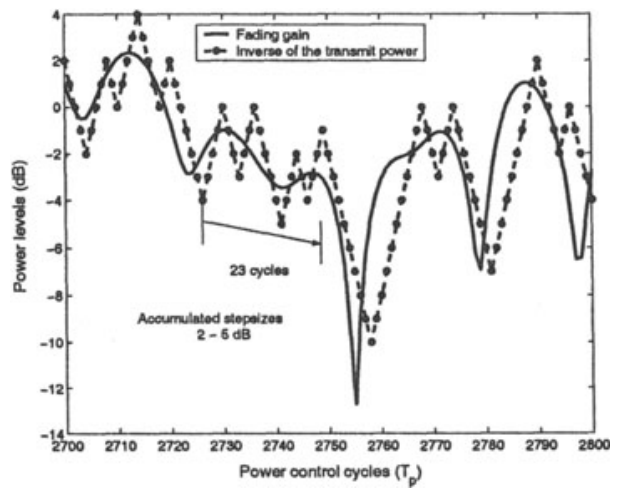

(b) Profile for fixed stepsize

Figure 4. Tracking capability of the adaptive stepsize power control, stepsize $=[1.35,1.0$, $0.74,0.5218,0.3737,0.2785,0.2] \mathrm{dB}$, and fixed stepsize power control at $1 \mathrm{~dB}$, with mobile speed $40 \mathrm{~km} /$ hour, $2 T_{\mathrm{p}}$ loop delay.

Figs. 4(a) and (b) illustrate the tracking ability of the adaptive stepsize and fixed stepsize TPC, respectively. In the figures, the transmit power is upshifted 
(aimed for $0 \mathrm{~dB}$ target received power) and reversed $\left(-P_{t}\right)$ in order to have a good visual effect. It is shown that adaptive stepsize TPC attempts to use curves to fit the variation of the fade, while the fixed stepsize TPC uses straight lines. The selection of the $\delta(n)$ is a tradeoff between the slope-overload distortion and granular noise. The main principle for the adjustment in Table 1 is to reduce the first few stepsizes for higher mobile speed, and to enlarge the first few stepsizes for lower mobile speed compared with the average. The reason is that for higher mobile speeds, the more frequently appeared fading valleys make the first few average stepsizes quite large. But in practical situations, it is difficult to fully counteract these valleys. As a result, the overall performance can be improved when we reduce these stepsizes appropriately to lower the granular noise. However, when the mobile speed is slower, properly increasing the first stepsize can compensate for the loop delay to a certain degree. It is noted that the results given in Table 1 have been obtained by test-and-trial based on the above principle. We believe that there should be a large space for the stepsize adjustment and the PCC memory length selection.

Table 3. Received power STD (dB) with optimal fixed stepsize and adaptive stepsize for different mobile speed, and the percentage gain in STD reduction.

\begin{tabular}{lccccccc}
\hline speed $(\mathrm{km} / \mathrm{h})$ & 20 & 25 & 30 & 35 & 40 & 45 & 50 \\
\hline Optimal fixed (dB) & 1.576 & 1.800 & 2.003 & 2.194 & 2.350 & 2.453 & 2.613 \\
Adap no adjust.(dB) & 1.526 & 1.685 & 1.875 & 1.989 & 2.067 & 2.111 & 2.234 \\
Adap w adjust.(dB) & 1.514 & 1.653 & 1.841 & 1.924 & 2.01 & 2.076 & 2.171 \\
\% Improvement & 3.95 & 8.16 & 8.15 & 12.31 & 14.59 & 15.42 & 16.92 \\
\hline
\end{tabular}

Table 4 lists the received power STD for the optimal fixed stepsize and adaptive stepsize TPC, and the percentage gain relative to the optimal fixed stepsize case. Significant improvement can be obtained, especially when the mobile speed is high.

\section{Conclusions}

An adaptive power control algorithm which minimizes the target power level and the standard deviation (STD) of the received power for DS-CDMA system is presented and evaluated. At the base station, an MMSE linear receiver is employed to demodulate the desired signal. At the mobile station, transmit power is updated with variable stepsize, which is generated based on PCC history and the estimated mobile speed.

Simulation results show that the MMSE iterative algorithm can converge to the target power level quickly. The proposed adaptive stepsize TPC exhibits a better tracking ability compared with the optimal fixed stepsize power control. It is conjectured that further improvement can be obtained through fine-tuning. 


\section{References}

[1] C. C. Lee and R. Steele, "Closed-loop power control in CDMA systems," Proc. of IEEE, , no. 4, pp. 231-239, Aug. 1996.

[2] H. J. Su and E. Geraniotis, "Adaptive closed-loop power control with quantized feedback and loop filtering," in Proc. IEEE Vehicular Technology Conf., , no. 1, pp. 76-86, Jan. 2002.

[3] S. Ariyavisitakul and L. F. Chang, "Signal and interference statistics of a CDMA system with feedback power control," IEEE Trans. Communications, pp. 1636-1634, Nov. 1993.

[4] F. Adachi, M. Sawahashi, and H. Suda, "Wideband DS-CDMA for next-generation mobile communications systems,” IEEE Communications Magazine, pp. 56-69, Sept. 1998.

[5] S. Nourizadeh, P. Taaghol, and R. Tafazolli, "A novel closed loop power control for UMTS," in 3G Mobile Communication Technologies, 2000, pp. 56-59.

[6] A. Chockalingam, P. Dietrich, L. B. Milstein, and R. R. Rao, "Performance of closedloop power control in DS-CDMA celluar systems," IEEE Trans. Vehicular Tech., pp. 774-789, Aug. 1998.

[7] J. W. Mark and S. Zhu, "Power control and rate allocation in multirate wideband CDMA systems," in Proc. IEEE Wireless Communications and Networking Conf. , pp. 168-172, 2000, (invited).

[8] J. T. Wu and E. Geraniotis, "Power control in multi-media CDMA networks," in Proc. IEEE Vehicular Technology Conf., pp. 789-793, 1995.

[9] L. C. Yun and D. G. Messerschmitt, "Power control for variable QoS on a CDMA channel," Proc. IEEE Military Communications Conf., pp. 178-182, Oct. 1994.

[10] U. Madhow and M. L. Honig, "MMSE interference suppresion for direct-sequence spread-spectrum CDMA," IEEE Trans. Communications, vol. 42, no. 12, pp. 3178-3188, Dec. 1994.

[11] S. Ulukus and R. D. Yates, "Adaptive Power Control with MMSE Multiuser Detectors," in Proc. IEEE Intl. Conf. Communications, pp. 361-365, 1997.

[12] R. D. Yates, "A framework for uplink power control in cellular radio systems," IEEE J. Select. Areas Communications, vol. 13, no. 7, pp. 1341-1347, Sept. 1995.

[13] P. S. Kumar and J. Holtzman, "Power control for a spread spectrum system with multiuser receivers," in Proc. IEEE Intl. Symposium on Personal, Indoor and Mobile Radio Communications, pp. 955-959, 1995. 